\title{
Effect of gravity convection on interface morphology during solidification
}

\author{
DUAN MengMeng ${ }^{\dagger}$, CHEN ChangLe ${ }^{\dagger}$, LI ZhanYao \& JIN QuanWei \\ Shaanxi Key Laboratory of Condensed Matter Structures and Properties, Northwestern Polytechnical \\ University, Xi'an 710072, China
}

An experimental apparatus consisting of a crystal growth room and a crystal growth observation system was developed for the study of the effect of the gravity convection perpendicular to the growth direction on the growth process by use of model alloy succinonitrile (SCN)-5wt\%ethanol. It was found that the convection improves the stability of the interface and causes the downstream alternation of the cell growth direction because of the dual effect of the Stokes force and the gravity. The second dendrite arm facing the flow comes into being earlier than that another side when the interface transforms cell to dendrite. Then the dendrite at the side facing the flow comes into being earlier. The second dendrite arm facing the flow grows faster and is more developed than that another side. In addition, the primary dendrite arm spacing increases and the dendrite tip radius decreases under the gravity convection.

gravity convection, solidification, interface morphology, model alloy

\section{Introduction}

The liquid flow is always existent in the process of solidification and the crystal growth, such as the liquid flow due to shrinkage of solid, interface flow, thermal convection and solute convection caused by the asymmetrical distribution of temperature and concentration, force flow and so on ${ }^{[1,2]}$. It is of significance to study the effect on solidification. Therefore, the international circles of solidification recognize that liquid flow is an important field in the modern solidification science.

Liquid flow has a great effect ${ }^{[3,4]}$ on the process of solidification and the quality of the grown crystal. It can change the temperature and concentration around the interface to influence the macrostructures and microstructures in solidification. For example, it can change the dendrite growth direction, accelerate the transformation of equiaxed dendrite to constrained columnar crystals, advance the dendritic unsymmetrical growth, change the dendrite arm spacing and so on ${ }^{[5-7]}$. However, the previous results have differences in some aspects, thus it is necessary to study

Received March 13, 2007; accepted May 15, 2007 doi: 10.1007/s11433-007-0052-8

${ }^{\dagger}$ Corresponding author (email: chenchl@nwpu.edu.cn or jsh810328@sohu.com)

Supported by the National Natural Science Foundation of China (Grant Nos. 50331040 and 60171043) 
the effect of flow on solidification.

In this study, the model alloy was chosen as the experimental study subject on account of its low melting point and transparency. We observed the directional solidification process by microscope accurately. Based on the experiment result, we analyzed the effect of the gravity convection on interface morphology during solidification in order to strengthen the understanding of the interaction between the liquid flow and solidification interface.

\section{Experimental method}

Succinonitrile (SCN)-5wt\%ethanol model alloy was chosen as the experimental study subject for its low melting entropy and transparency. Its Jackson coefficient is 0.97 , and it is non-facet growth crystal with a body-centered cube structure. Therefore, SCN and its alloy can be used to research metal simulatively.

Figures 1 and 2 are sketch diagrams of the model alloy growth room and the crystal growth observation system, respectively.
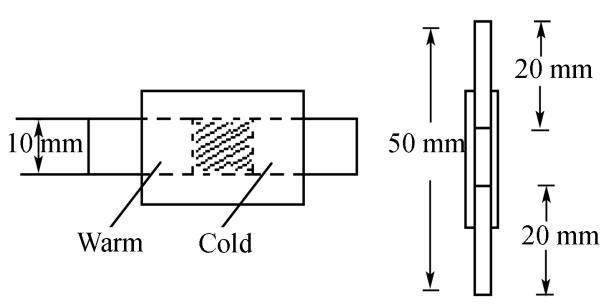

Figure 1 Schematic diagram of the crystal growth room.

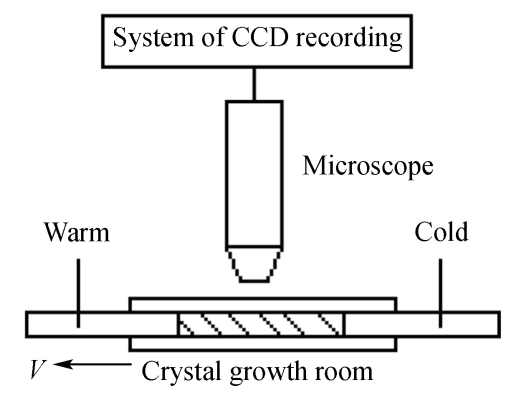

Figure 2 Schematic diagram of the crystal growth system.

The cool side of the crystal growth room was kept at a constant temperature. The hot side was heated by the circuit, which was made of exact systems of temperature controlling and recording. The temperature of the growth room changed from $10^{\circ} \mathrm{C}$ to $200^{\circ} \mathrm{C}$ and the fluctuation range was $\pm 0.1^{\circ} \mathrm{C}$. The interior cavity of the growth room was $500 \mu \mathrm{m}$.

The growth room filled with model alloy was located on the temperature gradient platform. The growth room pulled by the mechanism moved along the temperature gradient with a constant velocity of $50-100 \mu \mathrm{m} / \mathrm{s}$. We observed the crystal growth and flow distribution near the interface by microscope CKX41 and recorded them by CCD camera.

We fixed the crystal growth room on the microscope measuring platform to produce the gravity convection perpendicular to the growth direction by way of setting the room and microscope vertically (normal to the horizontal level). The bearing diagrams of the crystal growth room with and without the gravity convection are shown in Figures 3 and 4, respectively.

A number of tracer ions $(\Phi=2-5 \mu \mathrm{m})$ with the similar density as the model alloy were put into the room to demarcate the flow velocity.

\section{Experimental result and analysis}

\subsection{Effect of the gravity convection on flat interface}

Figure 5 is the morphology of the flat interface affected by the gravity convection, where the 


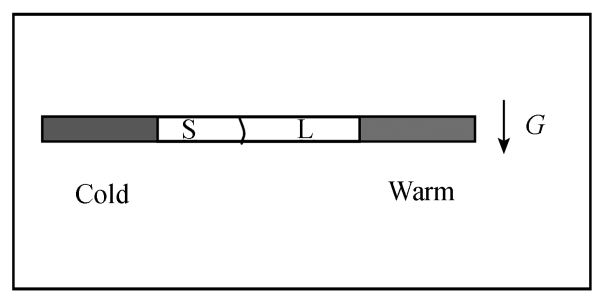

Figure 3 Bearing diagram of apparatus without convection (horizontal), observed from the top down.

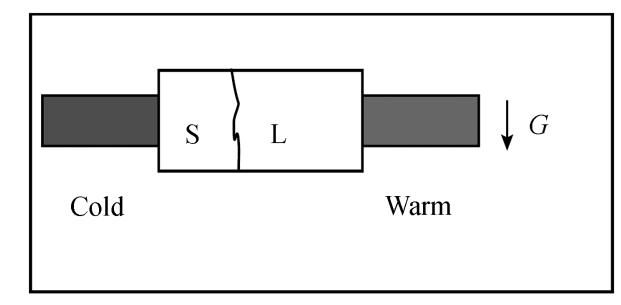

Figure 4 Bearing diagram of apparatus after convection (vertical), observed from outside to inside.

liquid velocity is $75 \mu \mathrm{m} / \mathrm{s}, t$ is the recording time, and $V_{0}$ is the growth velocity of the interface. The flange caused by fluctuation in the flat interface grows more slowly relative to the condition without the gravity convection.

The solute equilibrium partition coefficient $k_{0}$ of SCN-5wt\%ethanol alloy is less than 1 . When the crystal is growing, the solute is separated out and forms the solute boundary layer. The solute concentration of the boundary layer is distributed so unevenly that there is solute convection caused by the difference of the solute concentration. However, in our experiment, when the interface is flat, the solute convection is much less than the gravity convection. In other words, the effect of the gravity convection on the flat interface covers the solute convection.
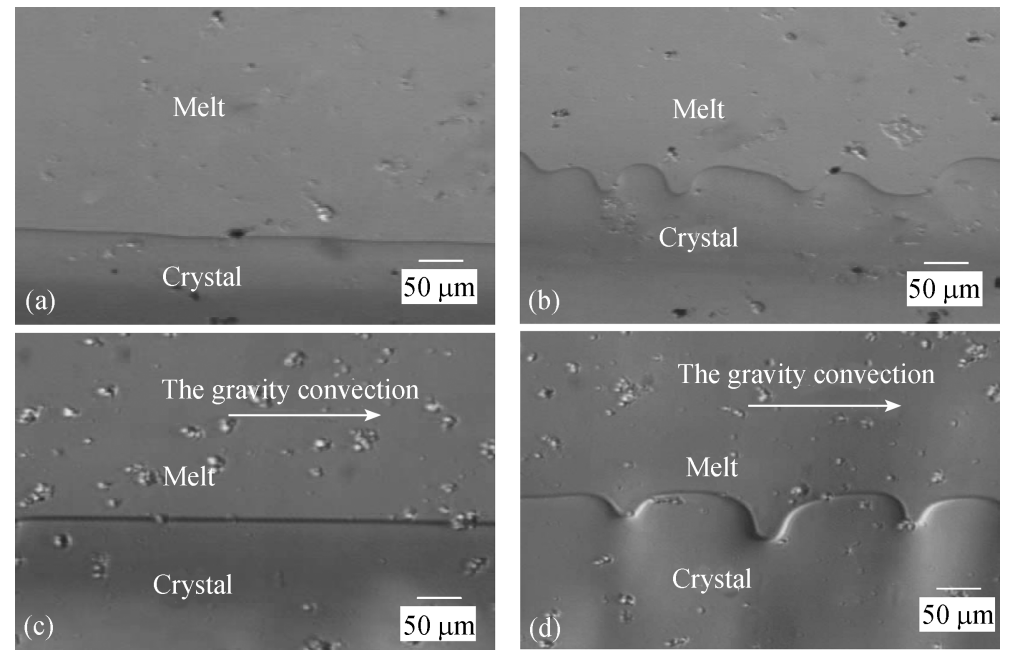

Figure 5 Effect of the gravity convection on the flat interface of SCN-5wt $\%$ ethanol, $G=3.72 \mathrm{~K} / \mathrm{mm}$. (a) $t=30 \mathrm{~s}$, without the gravity convection, $V_{0}=0.05 \mu \mathrm{m} / \mathrm{s}$; (b) $t=50 \mathrm{~s}$, without the gravity convection, $V_{0}=0.34 \mu \mathrm{m} / \mathrm{s}$; (c) $t=30 \mathrm{~s}$, after the gravity convection, $V_{0}=0.05 \mu \mathrm{m} / \mathrm{s}$; (d) $t=66 \mathrm{~s}$, after the gravity convection, $V_{0}=1.26 \mu \mathrm{m} / \mathrm{s}$.

In the process of the crystal growth, the area of the solute enriched in the solute boundary layer has a lower freezing point and is under the condition of constitutional supercooling. The flange caused by fluctuation in the flat interface grows owing to the constitutional supercooling, so the stabilization ${ }^{[8]}$ of the growth interface is destroyed. The interface is eroded by the gravity convection perpendicular to the growth direction, and then the solute which is separated out in solidification is carried away in time because of continuous erosion, so the flange caused by fluctuation in the flat interface grows more slowly.

We will analyze the conclusion mentioned above based on the theoretical calculations as follows.

The maximal growth velocity ${ }^{[9]}$ of flat to cell without the gravity convection is 


$$
V_{0 \max }=-\frac{G k_{0} D}{m C_{0}\left(1-k_{0}\right)},
$$

where $G$ is the temperature gradient, $k_{0}$ is the solute equilibrium partition coefficient, $m$ is the liquidus slope, $C_{0}$ is alloy composition, and $D$ is the diffusion coefficient.

In our experiment, for the SCN-5wt $\%$ ethanol, $G=3.72 \mathrm{~K} / \mathrm{mm}, k_{0}=0.2, D=1.89 \times 10^{-9} \mathrm{~m}^{2} / \mathrm{s}, C_{0}=5$, $m=-3.6 \mathrm{~K} / \mathrm{wt} \%$. According to the formula above, $V_{0 \max }=0.098 \mu \mathrm{m} / \mathrm{s}$ can be obtained.

When the growth velocity of the interface is $0.85 \mu \mathrm{m} / \mathrm{s}$, the solid/liquid interface is still flat under the effect of the gravity convection perpendicular to the growth direction. It is indicated that the gravity convection perpendicular to the growth direction improves the stability of the flat, which is consistent with the computation results of Mcfadden ${ }^{[10]}$ and Delves ${ }^{[11]}$ that the liquid flow parallel to the solid/liquid interface greatly improves the interface morphology stability.

\subsection{Effect of the gravity convection on cell interface}

Figure 6 is the picture we screened of the downstream alternation of cell due to the gravity convection. The velocity of the liquid flow is $75 \mu \mathrm{m} / \mathrm{s} . V_{0}$ is the growth velocity of the interface.
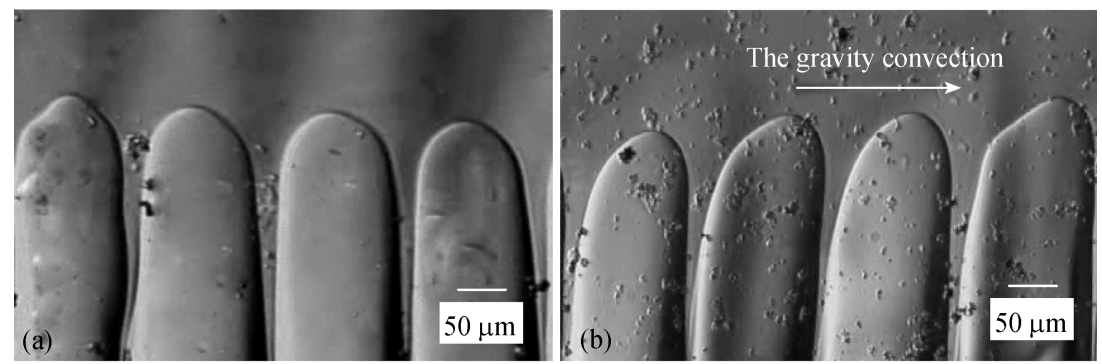

Figure 6 Effect of gravity convection on the cell interface of SCN-5wt\%ethanol, $G=3.72 \mathrm{~K} / \mathrm{mm}$. (a) Without the gravity convection; (b) after the gravity convection, $V_{0}=14.47 \mu \mathrm{m} / \mathrm{s}$.

Cell in directional solidification grows in the form of cell array. It is inaccurate to review the change of the growth morphology of a single cell in the flow. So we must consider not only the growth of the single cell, but also the interaction ${ }^{[12,13]}$ of the cell and its neighbor cell in time and space. Based on studying the cell array growth and analyzing the change of concentration of the cell tip, we can find out the mechanism of downstream alternation of the cell growth.

We will analyze the force of the cell tip in the flow to indicate the mechanism of downstream alternation of the cell.

The cell tip can be regarded as a hemisphere approximately, shown as Figure 7.

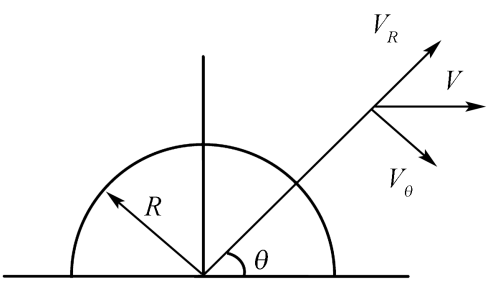

Figure 7 The ambient flow of the pellet at a low Reynolds number.

The Reynolds number in the flow is $R e=\frac{V x}{v}=8.347 \times 10^{-4} \ll 1$, where $x$ is the hemisphere 
diameter of the cell tip, $V$ is the velocity of the liquid flow, and $v$ is the kinematic viscosity. In our experiment, $x=28.94 \mu \mathrm{m}, V=75 \mu \mathrm{m} / \mathrm{s}, v=2.6 \times 10^{-6} \mathrm{~m}^{2} / \mathrm{s}$, thus the flow of the cell tip is a low Reynolds number one.

For our sample, $\rho$ is the melt density, $\rho=1.02 \mathrm{~g} / \mathrm{cm}^{3}$. According to the relation $\mu=v \rho$, $\mu=2.652 \times 10^{-3} \mathrm{~kg} /(\mathrm{m} \cdot \mathrm{s})$ can be obtained.

Because the inertia term can be ignored in a low Reynolds number flow of the cell tip, the motion Stokes equation in the flow can be written as follows:

$$
\left\{\begin{array}{l}
\nabla V=0, \\
\nabla P=\mu \nabla^{2} V+\rho g,
\end{array}\right.
$$

where $P$ is the press of the cell tip, and $g$ is the acceleration of the gravity.

According to the corresponding boundary conditions:

$$
\begin{aligned}
\text { at } r & =R, V_{r}=V_{\theta}=0 ; \\
\text { at } r=\infty, V_{r} & =V \cos \theta, \quad V_{\theta}=-V \sin \theta,
\end{aligned}
$$

the resolution is

$$
\left\{\begin{array}{l}
V_{R}=V \cos \theta\left(1-\frac{3 R}{2 r}+\frac{R^{3}}{2 r^{3}}\right), \\
V_{\theta}=-V \sin \theta\left(1-\frac{3 R}{4 r}-\frac{R^{3}}{4 r^{3}}\right), \\
P=P_{\infty}-\mu \frac{3 V R}{2 r^{2}} \cos \theta .
\end{array}\right.
$$

Based on the stress relation, the normal stress and the tangential stress in the sphere are

$$
\left\{\begin{array}{l}
f_{R}=\frac{3}{2} \mu V \frac{1}{R} \cos \theta-P_{\infty}, \\
f_{\theta}=-\frac{3}{2} \mu V \frac{1}{R} \sin \theta .
\end{array}\right.
$$

The force diagram of the cell tip under the gravity convection is shown in Figure 8 .

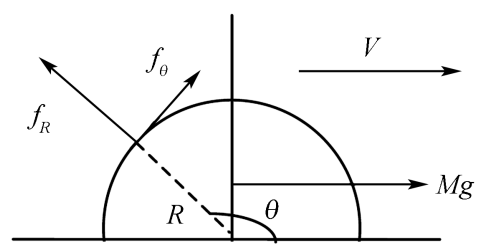

Figure 8 The force diagram of the cell tip under the gravity convection.

The cell tip in the viscosity fluid is under the Stokes force caused by the normal stress and the tangential stress as follows:

$$
F=\int_{0}^{\pi}\left(f_{\theta} \sin \theta-f_{R} \cos \theta\right) 2 \pi R^{2} \sin \theta \mathrm{d} \theta .
$$

Substituting eq. (1) into eq. (2) yields

$$
F=6 \pi v R V=0.5425 \times 10^{-10} \mathrm{~N} .
$$

The gravity of the cell tip is 


$$
G=M g=\rho \Omega g=0.6345 \times 10^{-10} \mathrm{~N} .
$$

The resultant force of the cell tip is

$$
F+G=1.18 \times 10^{-10} \mathrm{~N} .
$$

The gravity of the cell tip has the same direction as the Stokes force. Therefore, the combined effect of the thermodynamics factor, Stokes force and the gravity of the cell tip results in the downstream alternation of the cell interface.

\subsection{Effect of the gravity convection on cell to dendrite}

What we observed in the experiment is shown in Figure 9. The velocity of the liquid flow is 75 $\mu \mathrm{m} / \mathrm{s} . V_{0}$ is the growth velocity of the interface. It can be seen obviously that the fluctuation emerges in the interface facing the gravity convection (side B) earlier than that at another side (side A) for the dendrite $\mathrm{W}$. So the second dendrite arm at side B comes into being earlier than that at another side.

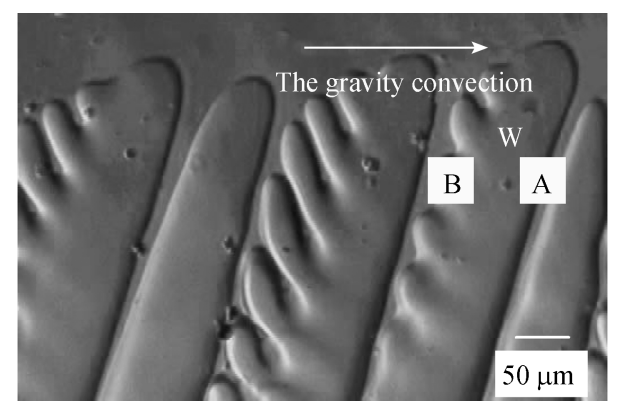

Figure 9 Effect of the gravity convection on cell to dendrite of SCN-5wt $\%$ ethanol, $V_{0}=21.32 \mu \mathrm{m} / \mathrm{s}, G=3.72 \mathrm{~K} / \mathrm{mm}$.

The solute concentration in the interface facing the gravity convection (side B) is lower than that at another side (side A) owing to the scouring action of the gravity convection. Then the supercooling degree in the interface of $\mathrm{B}$ is more than that in the interface of A when the interface transforms cell into dendrite. So the fluctuation in the interface of B can develop into dendrite more easily. We can see the second dendrite arm appearing in the cell interface facing the gravity convection when the interface transforms cell to dendrite. The interface opposite to the gravity convection is still a stable interface.

\subsection{Effect of the gravity convection on dendrite}

The change of dendrite under the gravity convection is shown as Figure 10. The velocity of the liquid flow is $75 \mu \mathrm{m} / \mathrm{s} . V_{0}$ is the growth velocity of the interface. As shown in Figure 10, the dendrite arm in one side of the cell comes out and enters into the groove of the neighbor cell. The solute is separated from the interface of the second dendrite arm. The solute in the groove of the neighbor cell is enriched so severely that the second arm of the neighbor cell in the side is restrained. Then it forms many dendrites ${ }^{[14]}$ of single side arms.

When the dendrite grows in the static melt, the second dendrite arms grow symmetrically along the primary dendrite arm crystal axis. When the direction of the liquid flow is perpendicular to the growth direction of the primary dendrite arm, the second dendrite arms facing the flow grow more fleetly and are more developed than those at another side.

The temperature fluctuation caused by the flow makes the dendrite tip uneven. The enriched solute, which is around the dendrite penetrating into the melt, is taken away by the flowing liquid in 


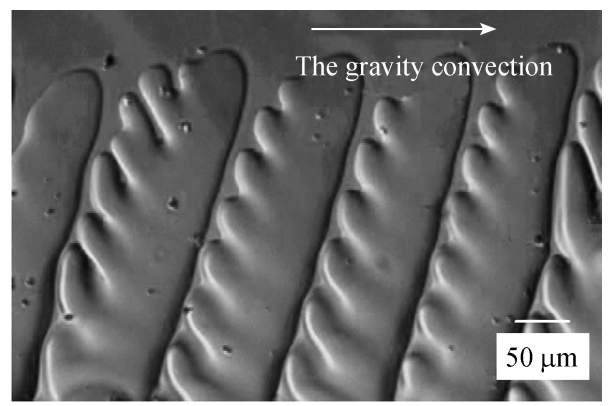

Figure 10 Effect of the gravity convection on dendrite of SCN-5wt $\%$ ethanol, $V_{0}=48.88 \mu \mathrm{m} / \mathrm{s}, G=3.72 \mathrm{~K} / \mathrm{mm}$.

time and causes severe thermal transmission in the interface. So the dendrite facing the flow grows fleetly. However, the dendrite upstream grows slowly until it stops growing finally.

The force of the dendrite tip is similar to that of the cell tip, so the dendrite growth direction is downstream alternation too.

The relation curves of Figure 11 are the changes of the pattern parametes (the primary dendrite arm spacing $\lambda$ and tip radius $R$ ) of dendrite both under the growth condition with and without the gravity convection at different growth velocity $V$.
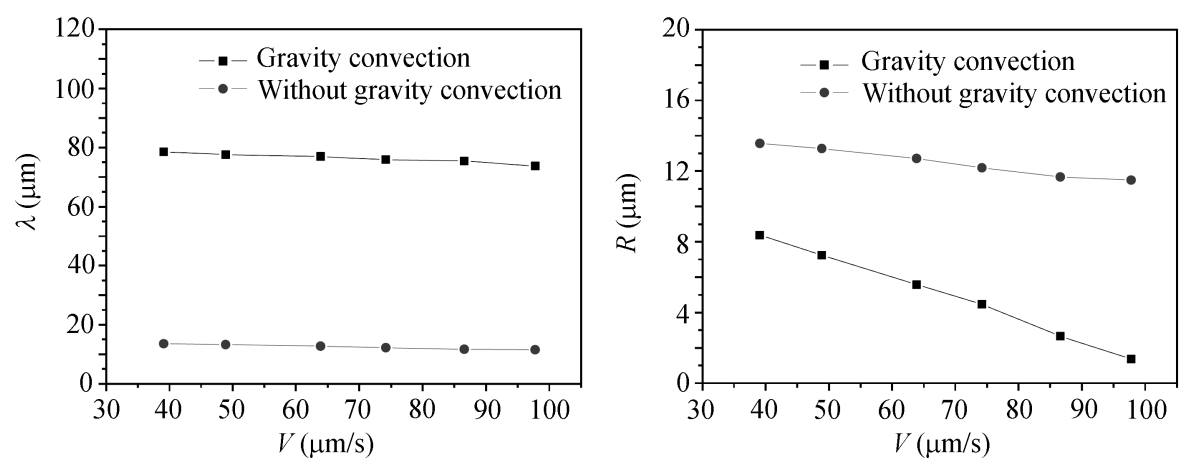

Figure 11 The relationship of the primary dendrite arm spacing and tip radius with a constant temperature gradient $G=3.72$ $\mathrm{K} / \mathrm{mm}$ at different growth velocities for $\mathrm{SCN}-5 \mathrm{wt} \%$ ethanol.

As shown in Figure 11, the primary arm spacing increases and the tip radius decreases under the gravity convection. The force supplied by the gravity convection accelerates the spacing adjustment of the strong dendrite and restrains the weak dendrite. The second dendrite arm facing the flow develops continuously and fleetly. Then the weak dendrite around the second arm is destroyed, so the primary arm spacing increases. For the tip radius, the increased dendrite growth velocity caused by the gravity convection reduces the tip radius according to the Interface Stability Theory; on the other hand, the equilibrium distribution of heat flux in the interface under the gravity convection makes the tip radius increased. However, the velocity of the gravity convection is so slow that the change of the tip radius as a result of equilibrium distribution of heat flux is smaller than the change due to the growth velocity. Then the tip radius reduces under the gravity convection.

\section{Conclusions}

Effect of the gravity convection perpendicular to the growth direction in solidification is summarized as follows: 
(1) The liquid flow improves the stability of the flat interface.

(2) The Stokes force and the gravity cause the downstream alternation of the cell growth direction.

(3) When the interface transforms cell to dendrite, the dendrite at the side facing the flow comes into being earlier.

(4) The second dendrite arm facing the flow grows faster and it is more developed than that at another side.

(5) The growth direction of dendrite is the downstream alternation which is the same as that of the cell.

(6) The primary dendrite arm spacing increases and the dendrite tip radius decreases.

1 Villers D, Platten J K. Coupled buoyancy and Marangoni convection in acetone: Experiments and comparison with numerical simulations. J Fluid Mech, 1992, 234: 487-510

2 Zhou B H, Liu Q S, Hu L, et al. Space experiments of thermocapillary convection in two-liquid layers. Sci China Ser E-Eng Mater Sci, 2002, 45(5): 552-560

3 McDonald R J, Hunt J D. Fluid motion through the partially solid regions of a casting and its importance in understanding A-type segregation. Trans Metall Soc AIME, 1969, 245: 1993-1995

4 Rosenberger F, Muller G. Interfacial transport in crystal growth, a parametric comparison of convective effects. J Cryst Growth, 1983, 65: 91-104

5 Shi H F, Xiao L, Liu Q. Simulation of effect of forced-convection on cellar and dendritic spacing in metal solidification. Nonferr Metal (in Chinese), 2004, 56(1): 21-23

6 Zhu C S, Wang Z P, Jing T, et al. Progress in convection effects on dendritic growth using phase-field method. Mater Rev (in Chinese), 2004, 18(12): 26-29

7 Beckermann C, Diepers H J, Steinbach I, et al. Modeling melt convection in phase-field simulations of solidification. J Comput Phys, 1999, 154: 468-496

8 Brattkus K, Davis S H. Flow-induced morphological instabilities: The rotating disc. J Cryst Growth, 1988, 87(4): 385 - 396

9 Lu D Y, Liu S, Huang T, et al. Influence of laminar flow parallel to liquid/solid interface on interface stability. Mater Sci Prog (in Chinese), 1992, 6(5): 400-403

10 Mcfadden G B, Coriell S R, Boisvert R F, et al. Morphological stability in the presence of fluid flow in the melt. Metall Trans A, 1984, 15: 2117-2124

11 Delves R T. Theory of Interface Stability in Crystal Growth. Pamplin B R, ed. London: Pergamon Press, 1974. 40-403

12 Mao Y J, Liu J, Zhou Y H. Study on downstream alternation of cell growth direction caused by melt flow. J Synth Cryst (in Chinese), 1997, 26(1): 47-51

13 Liu S, Lu D Y, Huang T, et al. Effect of melt flow on growth direction during constrained columnar crystal growth. J Northwest Polytech Univ (in Chinese), 1991, 9(1): 13-20

14 Zhu M F, Dai T, Lee S Y, et al. Modeling of dendritic growth in the presence of convection. Sci China Ser E-Eng Mater Sci, 2005, 48(3): $241-257$ 